\title{
Description of the F-16XL geometry and computational grids used in CAWAPI
}

\author{
O.J. Boelens ${ }^{1}$ \\ National Aerospace Laboratory NLR, P.O. Box 90502, 1006 BM, Amsterdam, The Netherlands \\ K.J. Badcock ${ }^{2}$ \\ CFD Laboratory, Department of Engineering, University of Liverpool, L697BZ, Liverpool, United Kingdom \\ S. Görtz ${ }^{3}$ \\ Royal Institute of Technology (KTH), 10044 Stokcholm, Sweden \\ S. Morton ${ }^{4}$ \\ United States Air Force SEEK EAGLE Office, Eglin AFB, 205 West D. Avenue, Suite 346, FL32542, United States \\ W. Fritz $z^{5}$ \\ EADS-MAS, OPEA31, 81633, Munich Germany \\ S.L. Karman Jr. ${ }^{6}$ \\ University of Tennessee at Chattanooga, Chattanooga, Tennessee, 37403, United States \\ T. Michal ${ }^{7}$ \\ The Boeing Company, St. Louis, Mo, United States \\ and \\ J.E. Lamar ${ }^{8}$ \\ NASA Langley Research Center, Hampton, VA 23681-2199, United States
}

\begin{abstract}
The objective of the Cranked-Arrow Wing Aerodynamics Project International (CAWAPI) was to allow a comprehensive validation of Computational Fluid Dynamics methods against the CAWAP flight database. A major part of this work involved the generation of high-quality computational grids. Prior to the grid generation an IGES file containing the air-tight geometry of the F-16XL aircraft was generated by a cooperation of the CAWAPI partners. Based on this geometry description both structured and unstructured grids have been generated. The baseline structured (multi-block) grid (and a family of derived grids) has been generated by the National Aerospace Laboratory NLR. Although the algorithms used by NLR had become available just before CAWAPI and thus only a limited experience with their application to such a complex configuration had been gained, a grid of good quality was generated well within four weeks. This time compared favourably with that required to produce the unstructured grids in CAWAPI. The baseline
\end{abstract}

\footnotetext{
${ }^{1}$ R\&D Engineer, Applied Computational Fluid Dynamics, Department of Flight Physics and Loads, Aerospace Vehicles Division, boelens@nlr.nl

${ }^{2}$ Professor, Department of Engineering, K.J.Badcock@liverpool.ac.uk

${ }^{3}$ Research Scientist, current affiliation: DLR, Lilienthalplatz 7, 38108 Braunschweig, Germany,

Stefan.Goertz@dlr.de

${ }^{4}$ Senior Research Scientist, Scott.Morton@eglin.af.mil

${ }^{5}$ R\&D Engineer, Aerodynamics and Methods., willy.fritz@eads.com

${ }^{6}$ Research Professor, Graduate School of Computational Engineering, Steve-Karman@utc.edu

${ }^{7}$ Technical Fellow, todd.r.michal@boeing.com

${ }^{8}$ CAWAP Principal Investigator, Configuration Aerodynamics Branch, MS 499, johnelamar@verizon.net
} 
all-tetrahedral and hybrid unstructured grids has been generated at NASA Langley Research Center and the USAFA, respectively. To provide more geometrical resolution, trimmed unstructured grids have been generated at EADS-MAS, the UTSimCenter, Boeing Phantom Works and KTH/FOI. All grids generated within the framework of CAWAPI will be discussed in the article. Both results obtained on the structured grids and the unstructured grids showed a significant improvement in agreement with flight test data in comparison with those obtained on the structured multi-block grid used during CAWAP.

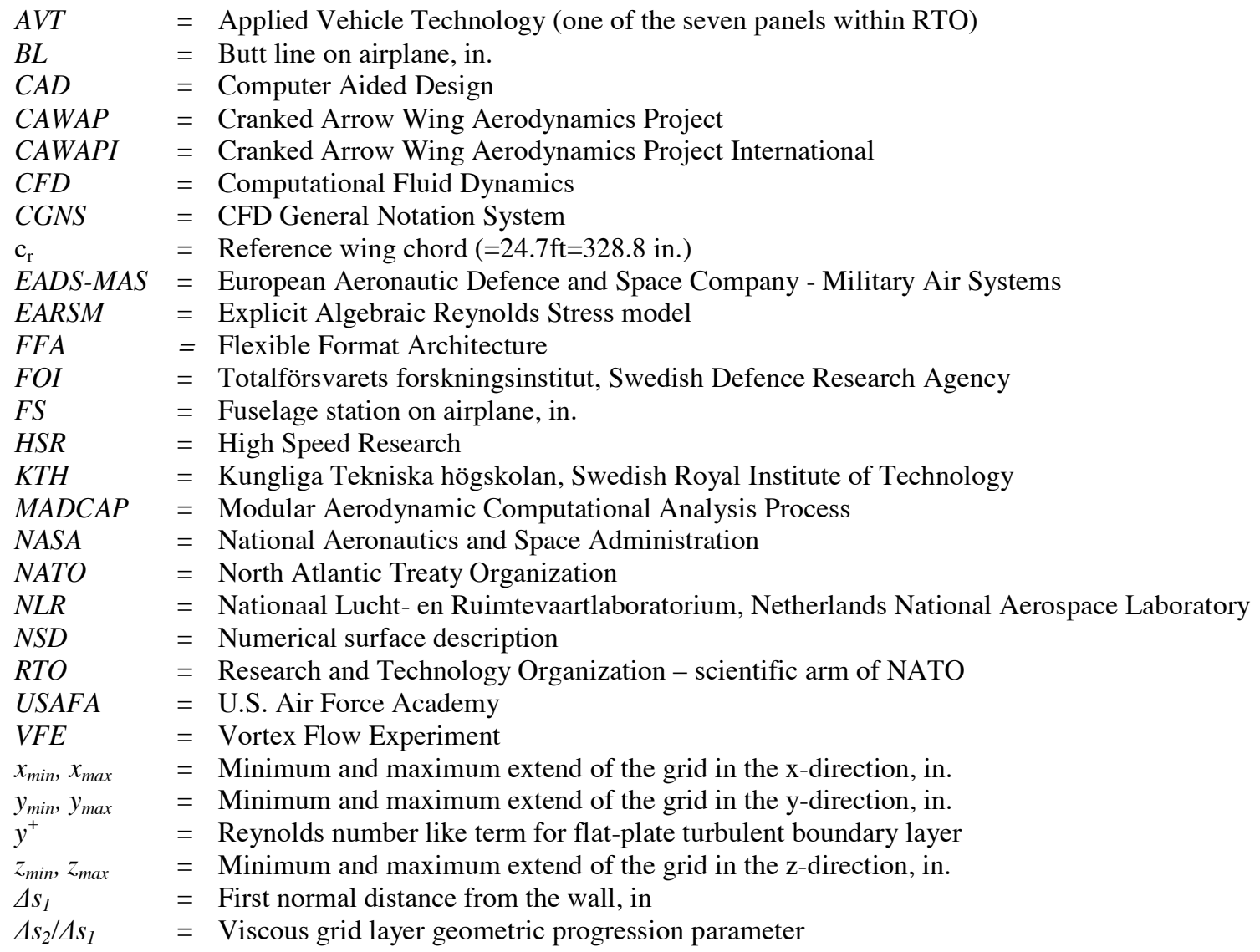

\section{Introduction}

THE Cranked-Arrow Wing Aerodynamics Project (CAWAP) provides the CFD community with an excellent database for validation and evaluation purposes [1][3]. The focus of this project was the understanding of the flow phenomena encountered on a cranked-arrow wing relevant to advanced fighter and transport aircraft. The subject of investigation was the F-16XL aircraft [1].

The Cranked-Arrow Wing Aerodynamics Project International (CAWAPI) [3] which was initiated by NASA was a follow-on project to the Cranked-Arrow Wing Aerodynamics Project. Along with the Vortex Flow Experiment 2 (VFE-2) [2] CAWAPI was incorporated under the NATO RTO working group AVT-113. The 
objective of the CAWAPI was to allow a comprehensive validation and evaluation of CFD methods against the CAWAP flight database [1][3].

Part of the work performed within CAWAPI involved the generation of high-quality computational grids. In order to allow high-quality grid generation, the available CAD geometry description of the F-16XL aircraft has been scrutinized. Issues encountered during this process are discussed in section II.

At the beginning of the project the working group members recognized the need to use common grids around this complex geometry to eliminate most of the uncertainties related to grid. The original plan was to have two common grids, one structured (multi-block) and one unstructured (tetrahedral). However, whereas all partners using structured CFD methods performed their simulation on a common structured multi-block grid generated at Netherlands National Aerospace Laboratory NLR (Sec. III), most partners using unstructured CFD methods have generated their own unstructured grid during the course of the project or have adapted existing grids (Sec. IV). A section with conclusions completes the article.

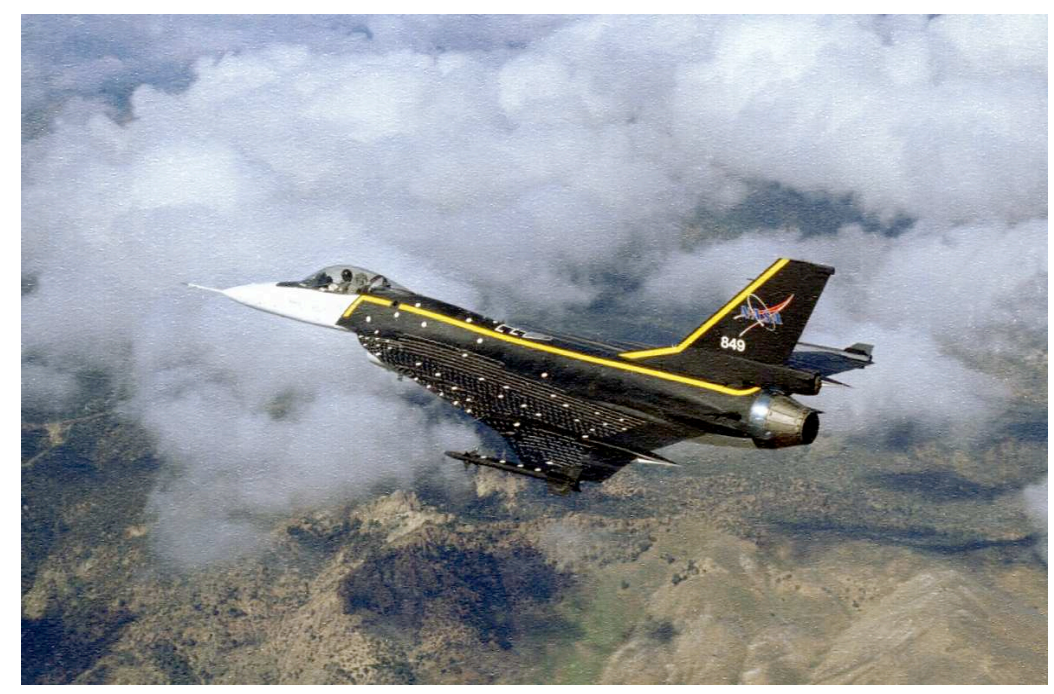

Fig. 1: F-16XL aircraft.

The present article only describes the generation of the high-quality computational grids (see also Table 1). The results obtained on both the structured and unstructured grids are described and discussed in the other articles of this Journal of Aircraft Special Section [3]-[7]. 


\section{Geometry description}

The F-16XL airplane [1][3] is a single-place, fighter-type prototype aircraft. The F-16XL airplane has a crankedarrow wing having a leading-edge sweep angle of $70^{\circ}$ inboard and $50^{\circ}$ outboard of the crank. During all CAWAP flight tests the aircraft was equipped with an air dam upstream of the actuator pod and wing-tip missiles. The airplane is shown in Fig. 1. For more details on the F-16XL airplane see Refs. [1] and [3].

In the framework of the Cranked-Arrow Wing Project International, the geometry of the F-16XL aircraft has been reconstructed using two surface descriptions, one from Lockheed Martin Aeronautics Company and one from NASA Langley. The latter was obtained by measuring the actual aircraft in the NASA hangar, where a numerical surface description (NSD) was obtained through photogrammetric targets. This measurement was performed in the framework of the HSR program [1][3]. Using both surface descriptions and additional CATIA models for the inlet up to the compressor face and for the nozzle up to the turbine face, an updated IGES file has been generated by Lockheed Martin Aeronautics Company. It should be noted that for the configuration used the control surfaces were not deflected. The updated IGES file contained a better characterization of the actual aircraft surfaces and leading edges, but was still not suitable for further grid generation purposes, since the geometry description contained multiple overlaying surfaces. This has been corrected at EADS-MAS, where a single set of describing surfaces was generated. The resulting surface description also included some refinements in the wing leading-edge region to improve future grid generation in this region. It was recognized by the CAWAPI members that this surface description still needed some further modifications to facilitate the generation of a structured grid. The following modifications have been applied:

- The gap between the launcher and the missile was closed. Other details of the missile, such as the fins, were unmodified.

- The gap between the nozzle and the trailing-edge flap was closed.

- The environmental control system (ECS) inlet was simplified.

- A step in the longitudinal progression of the nose-boom outer diameter was smoothed out.

These modifications were made at the NASA Langley Research Center. Finally, the modified surface description has been checked for air-tightness and corrected by EADS-MAS, where necessary, using the commercial CAD tool 'CADfix'. This IGES file containing the air-tight geometry description (see Fig. 2) has been used for both the structured and unstructured grid generation. 


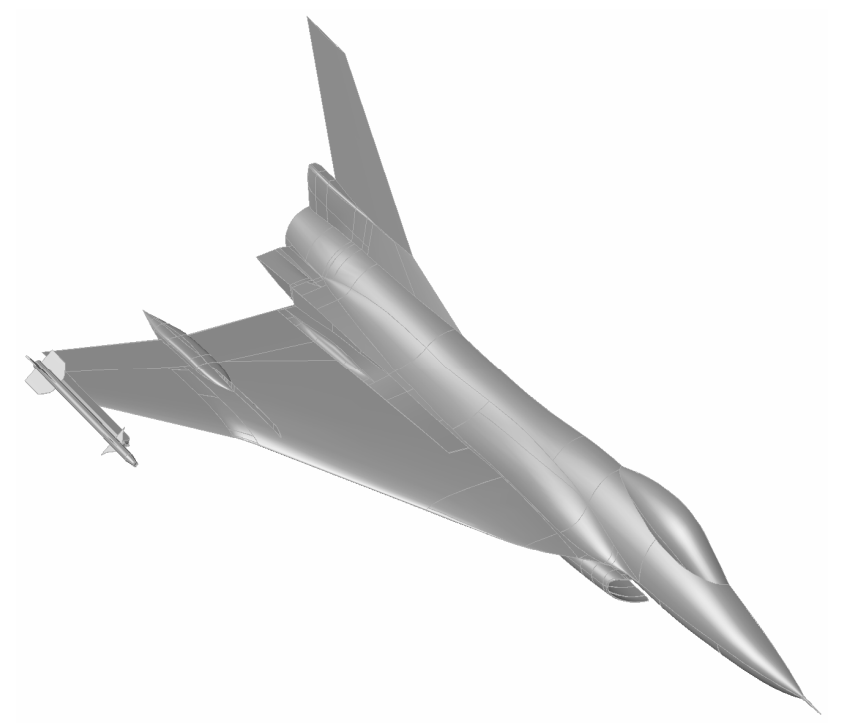

Fig. 2: Air-tight geometry description of the F-16XL aircraft.

\section{Structured multi-block grid}

\section{A. Background}

During CAWAP a structured multi-block grid had been generated at NASA Langley Research Center [1][3]. This grid was based on a prior IGES file and exhibited an average $\mathrm{y}^{+}$-value of 82 at a flight Reynolds number of around 40 million. Simulations on this grid [1] were performed using the 'wall function' option in the turbulence model. Since none of the participants to CAWAPI employed such a 'wall function' option in the turbulence model, it was decided that for CAWAPI purposes a new structured multi-block grid had to be generated.

The plan, as detailed in Ref. [8], was for "two members of the CAWAPI - one at the Netherlands National Aerospace Laboratory (NLR) and the other at the U.K. University of Glasgow (UGlasgow) -" to "collaborate in the development of the structured grid for their own use as well as for others. This is a risky endeavor even if the developers are co-located or on the same hall, but certainly more-so if they are in two different countries and having to rely on the Virtual Laboratory (VL) for all grid exchanges. The plan was for the NLR to produce the blocking strategy with implementation and for UGlasgow to adjust the grid spacing, as needed. Alternatively, NLR could produce and test the grid then UGlasgow would perform a second test on the grid before its general release to the facet. In either case, both would use and support the same grid file. For this problem, it turned out that the alternate plan was the one implemented due, in part, to the difficulties experienced with the transfer of large files...from this newly developed VL." 


\section{B. Grid generation algorithm}

The structured multi-block grid has been generated at the Netherlands National Aerospace Laboratory NLR using a Cartesian grid mapping technique. The (semi-automatic) grid generation algorithms have been developed at NLR and are part of NLR's ENFLOW flow simulation system [9]. Most of these algorithms had become available just before CAWAPI and had only been applied to a clean (no external loads) F-16 configuration. Being the first realistic case to which these tools were applied and bearing in mind that a limited experience with their use existed, it was estimated that six weeks would be needed to generate a complete structured multi-block grid around the halfspan full-scale model of the F-16XL aircraft.

The Cartesian grid generation technique used by the Netherlands Aerospace Laboratory NLR can be subdivided in the following steps:

1. Imagine/construct a Cartesian abstraction of the geometry description. In such an abstraction, the geometry including all details is represented by a set of Cartesian blocks. The abstraction of the half-span full-scale model of the F-16XL used in CAWAPI is shown in Fig. 3. In this figure, it can be observed that each fin of the wing tip missile for example is represented by a single block. Note furthermore that in this abstraction the engine duct and the nozzle have been closed.

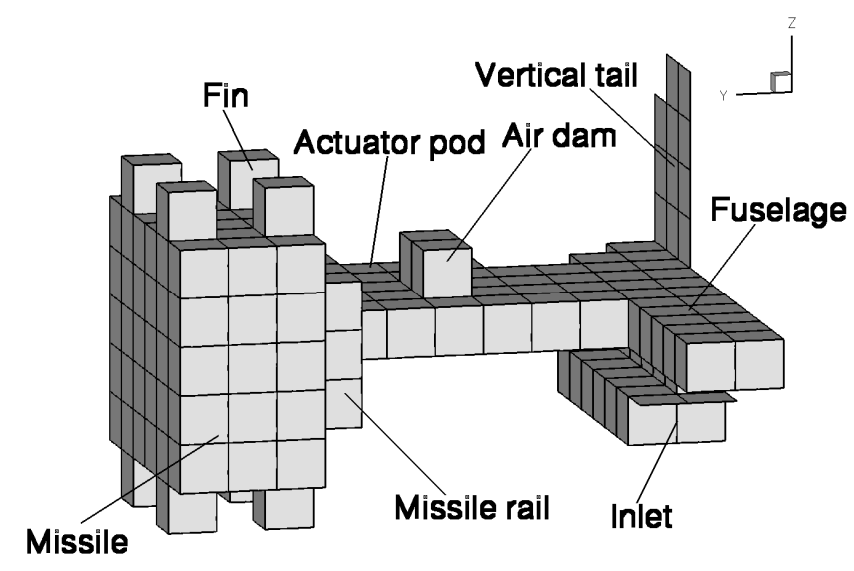

Fig. 3: Abstraction of the surface geometry for the half-span model of the F-16XL.

2. Project the abstraction onto the real geometry description. The projected abstraction of the half-span fullscale model of the F-16XL is shown in Fig. 4. 


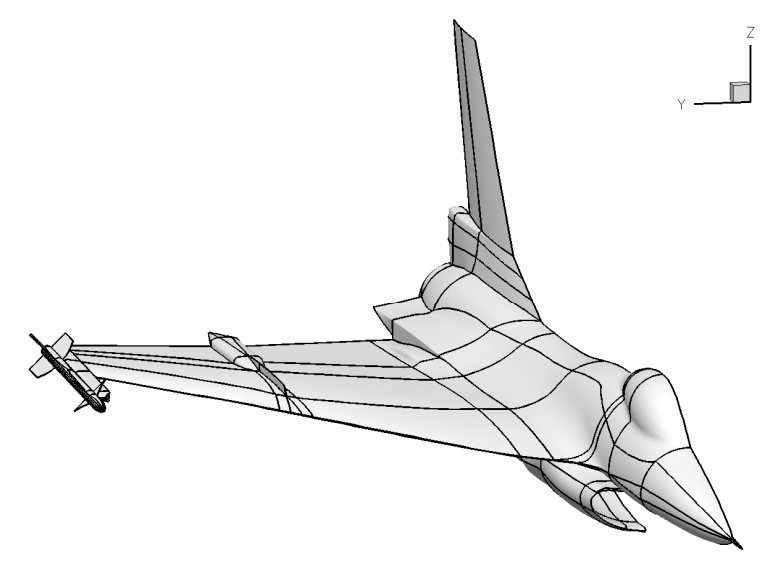

Fig. 4: Projected abstraction for the half-span model of the F-16XL.

3. Generate the so-called Navier-Stokes blocks. This first layer of blocks around the geometry including the engine duct and the nozzle is generated by a simple blow-up technique. The surface patches are translated along the outward normal to the geometry using the corners of the patches as control points. The algorithm used accounts for symmetry planes and only needs the off-set of the blocks as input. The generated layer of blocks has an O-O-type topology. During this step also the blocks to fill up the engine duct and the nozzle are inserted interactively.

4. Generate the field blocks in the Cartesian space. The faces of the Navier-Stokes blocks opposite to the geometry combined with the faces at the engine duct inlet and nozzle exit display the same Cartesian structure as the abstraction shown in Fig. 3. In the Cartesian space the field blocks are generated automatically. As is evident from Fig. 3, the blocks in the Cartesian space are simple cubical blocks.

5. Generate the field blocks in the physical space. The simple cubical blocks in the Cartesian space are automatically mapped to the physical space using a grid deformation technique [10]. The algorithm accounts for symmetry planes. Finally, so-called far-field blocks are added to the topology interactively. The far-field boundaries are located several reference wing chords away from the model.

6. Set the (Euler) grid dimensions. Each edge is assigned a grid dimension. The minimum number of cells used along an edge is eight, to ensure three levels of multi-grid. In the Navier-Stokes blocks, eight cells were used in the surface normal direction.

7. Automatically connect the edges. The grid spacing in the grid is set automatically. For each set of adjoining edges the grid point density is adjusted such that a smooth transition of the grid is obtained. In general, this 
means that the grid point density of the edge with the larger grid spacing is linked to that of the edge with the smaller grid spacing.

8. Improve the grid quality by an elliptical smoothing algorithm. An elliptical smoothing algorithm is applied to the grid. As a result of this algorithm the quality in terms of grid smoothness is improved significantly.

9. Increase the resolution in the Navier-Stokes blocks. To provide for sufficient boundary layer resolution, the number of grid points in the surface normal direction in the Navier-Stokes blocks is increased. In addition a redistribution of the grid points with a specified stretching away from the geometry is applied. The algorithm used accounts for a smooth transition to the grid in the outer blocks.

Within NLR's ENFLOW flow simulation system [9] further algorithms exist to:

- Merge blocks within a grid to reduce the total number of blocks.

- Mirror a grid with respect to a symmetry plane to obtain a full-configuration grid from a half-configuration grid.

- Convert the grid from NLR's native ENFLOW format to several other formats, such as Plot3D or CGNS [11].

The characteristics of the structured grid obtained using this Cartesian grid mapping technique are described in the next section.

Instead of the six weeks estimated prior to the project, the structured grid has been generated well within four weeks, including some further development of the grid generation algorithms.

\section{Characteristics of the structured multi-block grid}

During the grid generation process the following modifications to the surface description of the F-16XL aircraft have been made to further facilitate the generation of a multi-block structured grid:

- A small 'step' or 'plate' on the wing upper surface was removed.

- The end part of the vertical tail base was slightly rounded off.

It is, however, expected that these modifications do not influence the simulated flow significantly.

The following family of structured grids has been used in CAWAPI: 
- The baseline structured grid around the half-span full-scale model of the F-16XL consisting of 1903 blocks, $14,750,720$ grid cells and 17,014,119 grid points.

- The baseline structured grid with the far-field blocks divided into smaller blocks so that only a one-to-one connection between block faces exists. This version was used by the University of Liverpool.

- The baseline structured grid with a reduced number of blocks. The first merging step was performed at Netherlands National Aerospace Laboratory NLR reducing the number of blocks from 1903 to 216. A further small reduction was accomplished at NASA Langley Research Center which yielded a grid with only 200 blocks.

- A structured grid around the full-scale model of the F-16XL consisting of 3806 blocks, 29,501,440 grid cells and 34,028,238 grid points. This grid has been generated by mirroring the baseline structured grid around the half-span full-scale model of the F-16XL with respect to the symmetry plane. This grid has only been used by Netherlands National Aerospace Laboratory NLR

Some further details of the baseline structured grid around the half-span full-scale model of the F-16XL are summarized in Table 1.

The upper surface grid of the structured multi-block grid is shown in Fig. 5. In Fig. 6, the grid is shown in both a plane approximately normal to the flow direction (FS is constant) and in a plane approximately parallel to the flow direction (BL is constant). 
Table 1: Overview of the baseline structured and unstructured grids employed during CAWAPI

\begin{tabular}{|c|c|c|c|c|c|c|}
\hline Grid & Generated by & Grid generation tool & $\begin{array}{l}\text { Grid described } \\
\text { in section }\end{array}$ & Grid size & $\begin{array}{l}\text { Boundary layer } \\
\text { settings }\end{array}$ & $\begin{array}{l}\text { Results shown in } \\
\text { Ref. }\end{array}$ \\
\hline Baseline structured & $\begin{array}{l}\text { National Aerospace } \\
\text { Laboratory NLR }\end{array}$ & $\begin{array}{l}\text { In-house developed, part of } \\
\text { NLR's ENFLOW flow } \\
\text { simulation system }\end{array}$ & III & $\begin{array}{l}1903 \text { blocks } \\
14,750,720 \text { grid cells } \\
17,014,119 \text { grid points }\end{array}$ & $\begin{array}{l}\Delta \mathrm{s}_{1}=7.910^{-7} \mathrm{c}_{\mathrm{r}} \\
\Delta \mathrm{s}_{2} / \Delta \mathrm{s}_{1}=1.1\end{array}$ & [4] [7] \\
\hline $\begin{array}{l}\text { Baseline all- } \\
\text { tetrahedral } \\
\text { unstructured }\end{array}$ & $\begin{array}{l}\text { NASA Langley } \\
\text { Research Center }\end{array}$ & GridTool, VGRIDns & IV.A & $\begin{array}{l}2,534,132 \text { nodes } \\
14,802,429 \text { tetrahedra }\end{array}$ & - & {$[5][7]$} \\
\hline $\begin{array}{l}\text { Baseline hybrid } \\
\text { unstructured }\end{array}$ & USAFA & Blacksmith & IV.A & $\begin{array}{l}2,535,842 \text { nodes } \\
1,442,394 \text { prisms }(9 \\
\text { layers }) \\
10,482,709 \text { tetrahedra }\end{array}$ & $\begin{array}{l}\Delta \mathrm{s}_{1}=6.610^{-6} \mathrm{c}_{\mathrm{r}} \\
\Delta \mathrm{s}_{2} / \Delta \mathrm{s}_{1}=1.2\end{array}$ & [5] [7] \\
\hline $\begin{array}{l}\text { EADS trimmed } \\
\text { hybrid unstructured }\end{array}$ & EADS-MAS & $\begin{array}{l}\text { CentaurSoft, adaptation } \\
\text { algorithm in DLR-TAU code }\end{array}$ & IV.B.1 & $\begin{array}{l}10,496,522 \text { nodes } \\
\sim 15,600,000 \text { prisms }(29 \\
\text { layers) } \\
\sim 13,500,000 \text { tetrahedra }\end{array}$ & $\begin{array}{l}\Delta \mathrm{s}_{1}=4.810^{-7} \mathrm{c}_{\mathrm{r}} \\
\Delta \mathrm{s}_{2} / \Delta \mathrm{s}_{1}=1.3\end{array}$ & [6] [7] \\
\hline $\begin{array}{l}\text { UTSimCenter } \\
\text { trimmed hybrid } \\
\text { unstructured }\end{array}$ & $\begin{array}{l}\text { University of } \\
\text { Chattanooga SimCenter }\end{array}$ & Gridgen, in-house developed & IV.B.2 & $\begin{array}{l}13,906,708 \text { nodes } \\
15,770,674 \text { prisms }(25 \\
\text { layers }) \\
166,230 \text { pyramids } \\
32,395,936 \text { tetrahedra }\end{array}$ & $\begin{array}{l}\mathrm{y}^{+} \sim 1 \\
\Delta \mathrm{s}_{2} / \Delta \mathrm{s}_{1}=1.15 \\
\text { geometric } \\
\text { growth rate: } \\
1.02\end{array}$ & {$[6][7]$} \\
\hline $\begin{array}{l}\text { Boeing trimmed } \\
\text { hybrid unstructured }\end{array}$ & Boeing Phantom Works & MADCAP & IV.B.3 & $\begin{array}{l}\sim 19,300,000 \text { cells } \\
15 \text { prismatic layers }\end{array}$ & $\begin{array}{l}\Delta \mathrm{s}_{1}=9.110^{-7} \mathrm{c}_{\mathrm{r}} \\
\Delta \mathrm{s}_{2} / \Delta \mathrm{s}_{1}=1.2\end{array}$ & [6] [7] \\
\hline
\end{tabular}




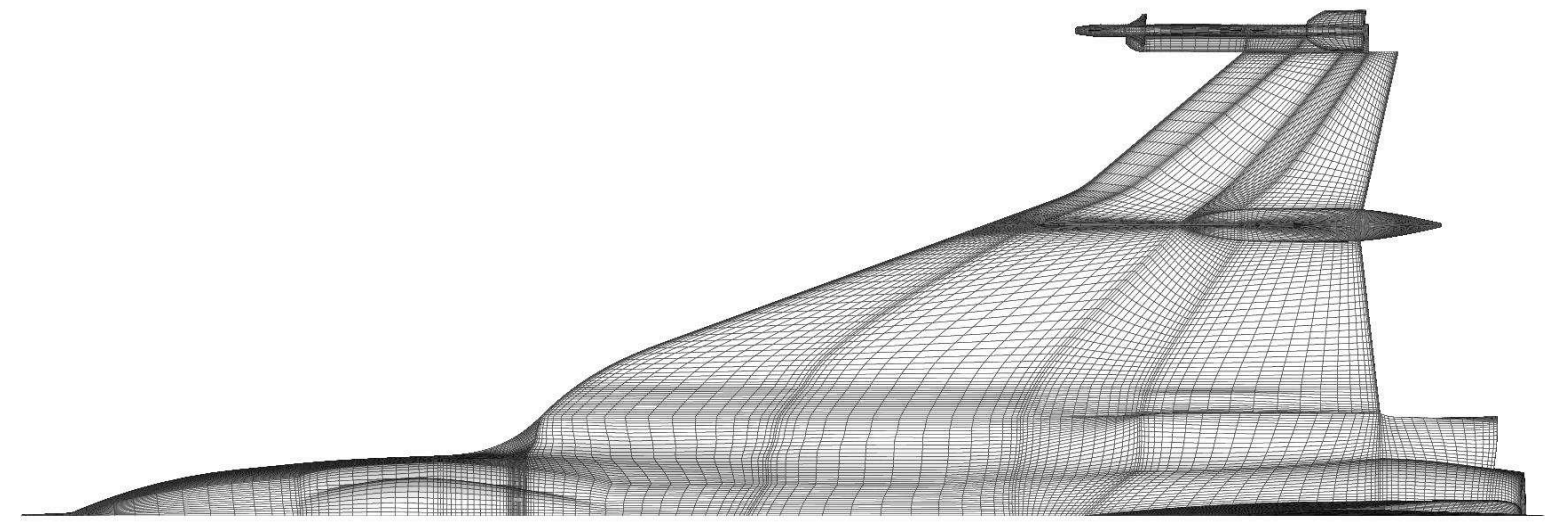

Fig. 5: Upper surface grid for the structured multi-block grid for the half-span model of the F-16XL.
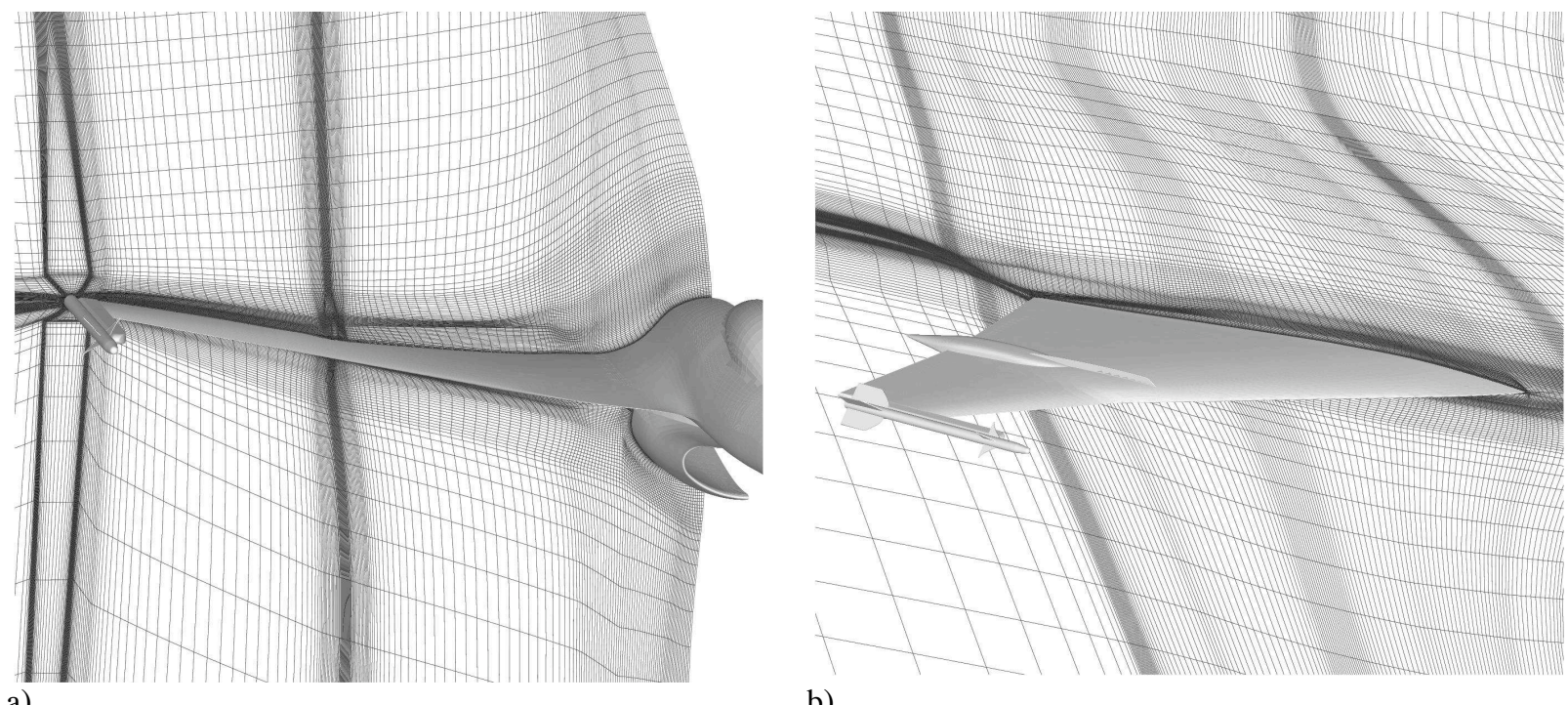

b)

Fig. 6: Grid planes showing the grid density off the aircraft surface in a) a plane approximately normal to the flow direction (FS is constant) and b) a plane approximately parallel to the flow direction (BL is constant).

A good resolution of the boundary layer requires the grid to be clustered in the direction normal to the surface with the spacing of the first grid point off the wall to be well within the laminar sub-layer of the boundary layer. For turbulent flows, the first point off the wall should exhibit a $\mathrm{y}^{+}$-value of aprroximately one. The resulting $y^{+}$ distribution over the upper surface for the structured multi-block grid is shown in Fig. 7 for flight condition 19. From this figure, it is evident that the grid spacing normal to the surface results in $y^{+}$-values of approximately one. 


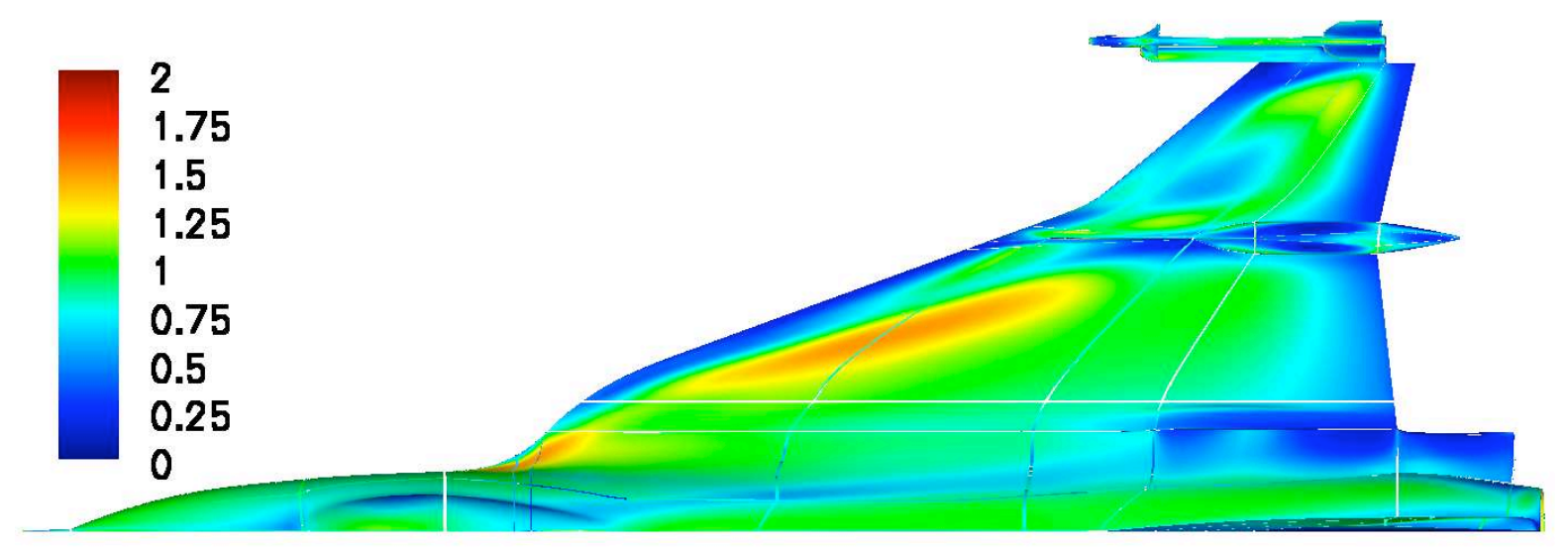

Fig. 7: Levels of $y^{+}$on the upper surface for the structured multi-block grid for flight condition $19\left(\alpha=11.85^{\circ}\right.$, $M=0.360$ and $R e=46.8010^{6}$ ). Result obtained using the TNT k- $\omega$ turbulence model with correction for vortical flows.

The grid converted to Plot3D format was uploaded to the Virtual Laboratory (VL) [3][8] at NASA Langley Research Center to be used by other researchers in CAWAPI.

\section{Unstructured grids}

During CAWAP no unstructured grid had been generated. Within the framework of CAWAPI the plan was to generate a common unstructured (tetrahedral) grid. However, as discussed in Sec. I, most partners using unstructured CFD methods have generated their own unstructured grid during the course of CAWAPI or have adapted existing grids (see Table 1). Baseline all-tetrahedral and hybrid unstructured grids (Sec. IV.A) have been generated at NASA Langley Research Center and USAFA, respectively. Trimmed unstructured grids have been generated at EADS-MAS (Sec. IV.B.1), the UTSimCenter (Sec. IV.B.2), and Boeing Phantom Works (Sec. IV.B.3). In addition, a series of highly-adapted grids, both Euler and Navier-Stokes, has been been generated at KTH/FOI. These grids that have been used in an extensive study of flight condition 70, are discussed in [7].

\section{A. Baseline unstructured grids}

At the NASA Langley Research Center a baseline unstructured all-tetrahedral viscous grid with 2,534,132 nodes, corresponding to $14,802,429$ cells, has been generated around the half-span full-scale model of the F-16XL using the grid generation packages GridTool [12] and VGRIDns [13].

This grid has been converted to a hybrid baseline unstructured grid in Cobalt [14] format at USAFA using the commercial grid management utility Blacksmith from Cobalt Solutions, LLC. Blacksmith reduced the cell count to a 
total of $11,928,103$, corresponding to $2,535,842$ nodes, by combining highly stretched tetrahedral cells into prismatic cells. The program generated nine layers of prismatic cells, corresponding to 1,442,394 prisms. The reason the grid had only nine prismatic layers is that pyramids would be needed as 'end caps' for layers that are not complete. Rather than adding another cell type it was decided to accept those nine layers. The transition between the prismatic layers and the tetrahedral grid is very smooth. The surface of the half-span full-scale model of the F-16XL is discretized with 160,266 triangular elements. The upper surface grid is shown in Fig. 8.

For the hybrid baseline unstructured grid the spacing of the first grid point normal to the solid wall is $6.6 \times 10^{-6} \mathrm{c}_{\mathrm{r}}$. Away from the wall, the spacing increases by a ratio $\Delta \mathrm{s}_{2} / \Delta \mathrm{s}_{1}$ of 1.2 . The resulting $\mathrm{y}^{+}$distribution over the upper surface of the aircraft model is shown in Fig. 9 for flight condition 19. It can be seen that the grid spacing normal to the surface led to an average $\mathrm{y}^{+}$-value of one and a maximum $\mathrm{y}^{+}$-value of about two under the primary wing vortex, demonstrating that the grid is fine enough at the wall boundaries.

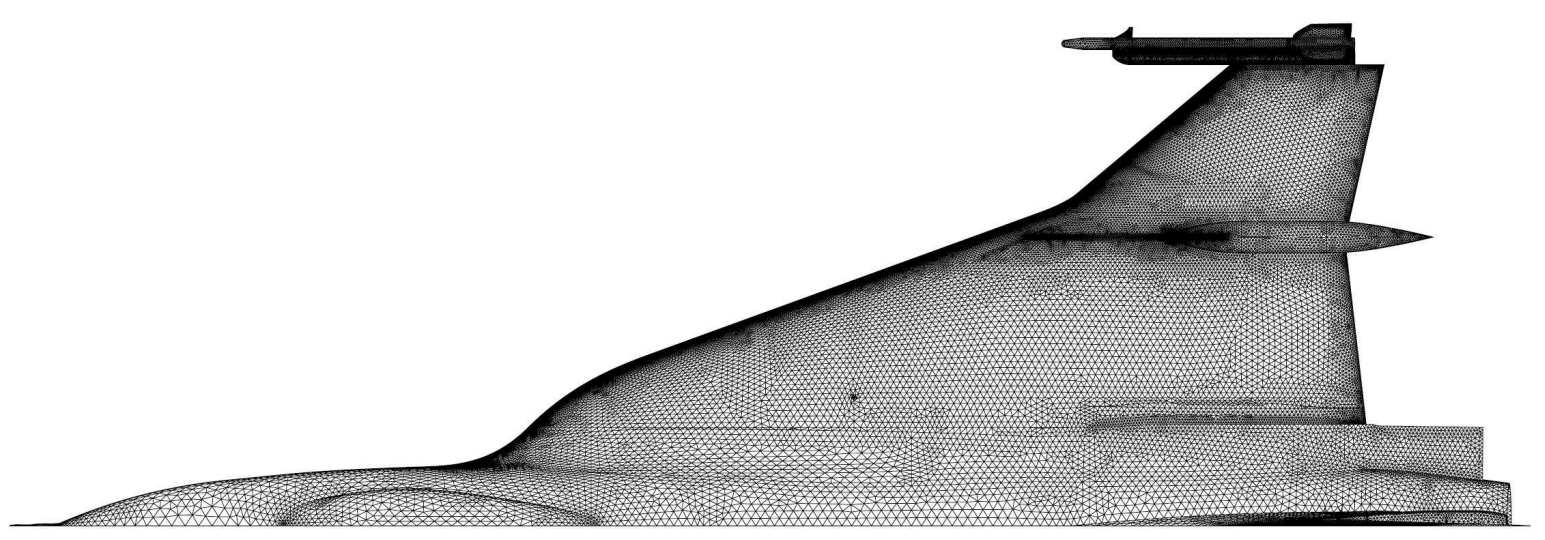

Fig. 8: Surface grid of the hybrid baseline unstructured grid for the half-span model of the F-16XL (160,266 faces).

The engine duct is meshed all the way to the compressor face, while the nozzle is meshed from the engine mixing plane, see Fig. 10. The grid density off the aircraft surface is shown in Fig. 11, which depicts a wrinkly cutting plane through the grid at FS496 (fuselage station on airplane in inches, positive aft), close to the trailing edge. 


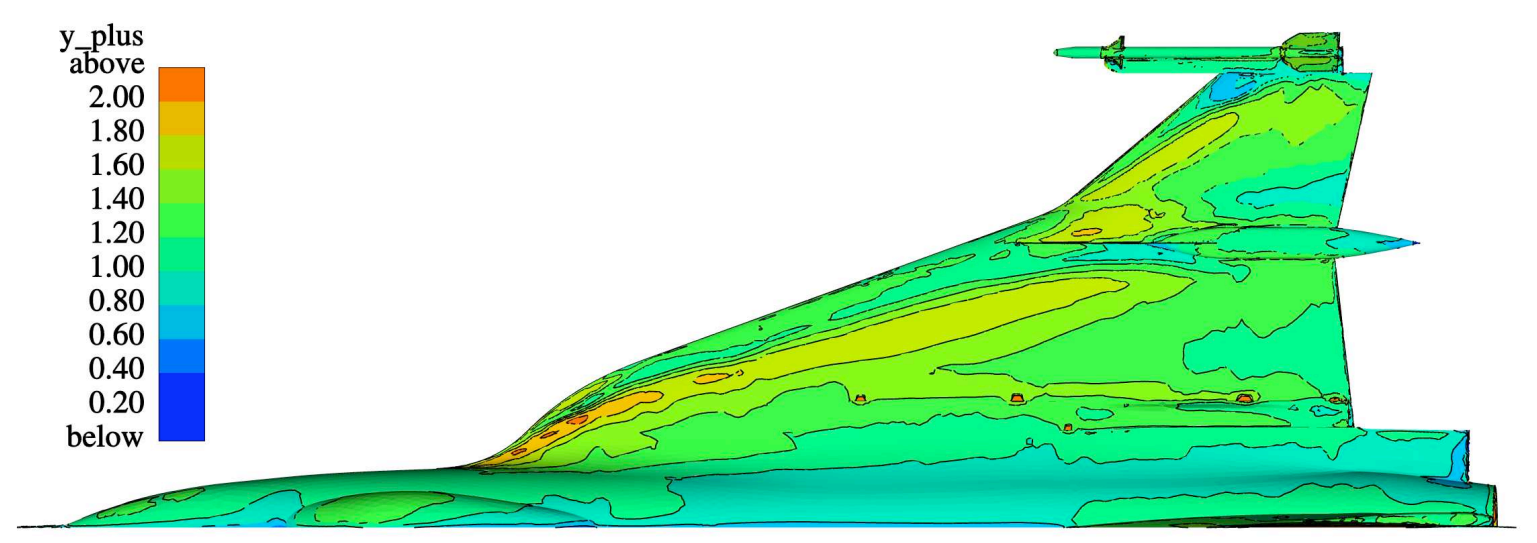

Fig. 9: Levels of $y^{+}$on the upper surface for the hybrid baseline unstructured grid for flight condition 19 $\left(\alpha=11.85^{\circ}, M=0.360\right.$ and $\left.R e=46.8010^{6}\right)$. Result obtained using the EARSM turbulence model.

Next, researcher at KTH/FOI converted the hybrid grid from Cobalt format to the "Flexible Format Architecture" (FFA) [15], the native format of the Swedish CFD code Edge [16]. In this conversion step, all grid dimensions were converted from inches to meters.

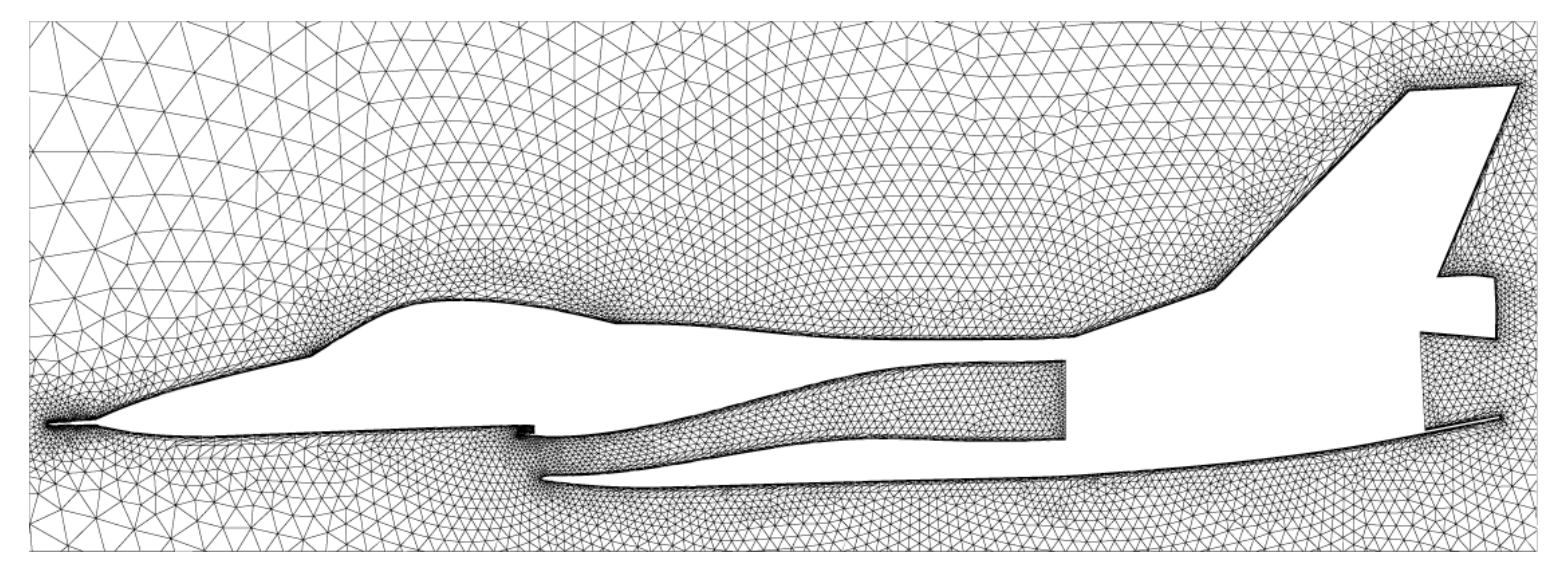

Fig. 10: Symmetry plane of the hybrid baseline unstructured grid showing the grid in the engine duct and nozzle.

Finally, KTH/FOI researchers converted the FFA-format grid to the CFD General Notation System (CGNS) [11] library version 2.3. The resulting CGNS file was uploaded to the Virtual Laboratory (VL) [3][8] at NASA Langley Research Center to be used by other researchers in CAWAPI. 


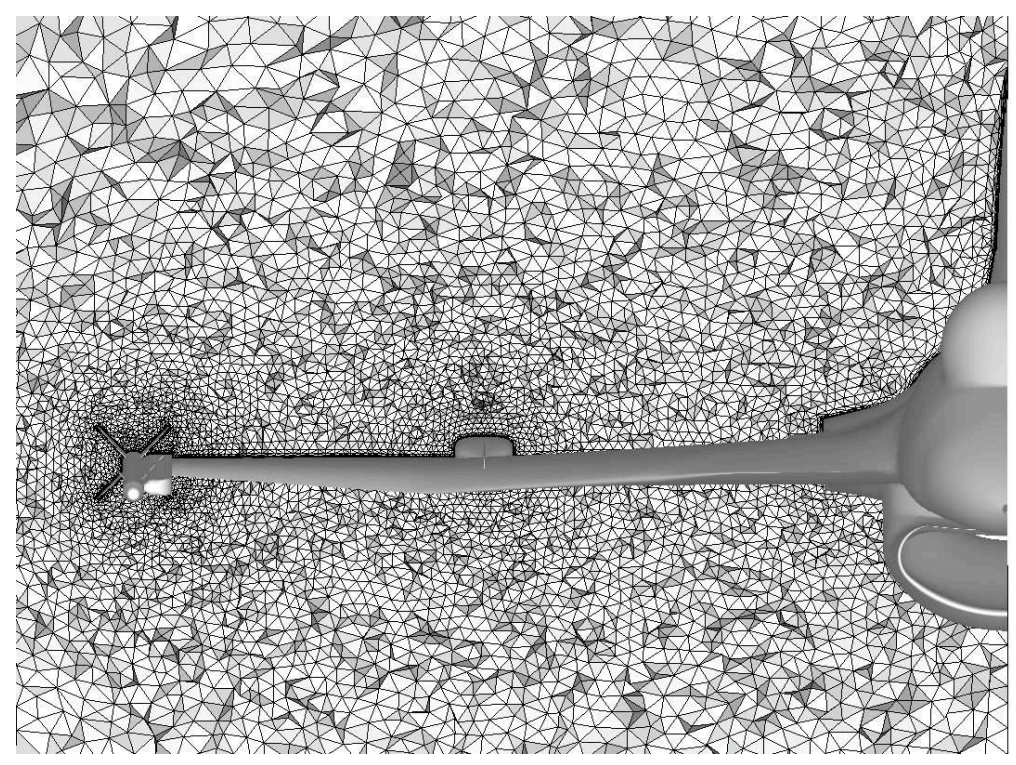

Fig. 11: Wrinkly cutting plane of the hybrid baseline unstructured grid at FS496 showing the grid density off the aircraft surface close to the trailing edge of the wing.

\section{B. Trimmed unstructured grids}

\section{EADS-MAS}

At EADS-MAS the grid adaptation technique, which is included in the DLR-TAU code [17], has been used for the CAWAPI CFD calculations. Starting point was an initial grid, which subsequently is adapted four times during the flow calculations. The initial grid has been generated using the commercial CentaurSoft grid generator, which enables the generation of hybrid grids with minimal user interaction. Starting from the air-tight geometry description, the grid generation process is split up into surface triangulation, prismatic grid generation and tetrahedral grid generation. Point clustering is achieved by automatic clustering based on geometric features and by user-controlled clustering placing so-called sources. This user-controlled clustering has been used for a rough adaptation of the grid to the expected vortical flow structure. The surface triangulation works patch-oriented, which results in a high resolution of all small surface patches. This high resolution however is not always needed. The geometry description of the F-16XL aircraft contains several such mini-patches. As the adaptation algorithm of the DLR-TAU code uses the surface grid as geometry base, the surface triangulation of this initial grid was already relative fine to ensure a sufficient resolution of all geometric details. The tetrahedral grid however was kept somewhat coarse and was expected to be refined by the adaptation. 
The resulting initial grid is a hybrid grid with 10,496,522 nodes in total for the half-span full-scale model of the F-16XL. It has a prismatic layer of 15.6 million prisms in the near wall region and 13.5 million tetrahedra in the outer region. The thickness of the first prismatic layer is $4.8 \times 10^{-7} \mathrm{c}_{\mathrm{r}}$ and a geometric progression parameter $\Delta \mathrm{s}_{2} / \Delta \mathrm{s}_{1}$ of 1.3 is used for the other 29 viscous layers. In critical regions the prismatic layers are chopped and transition elements such as pyramids and tetrahedra are created. The surface of the aircraft is resolved by 749,742 triangles.

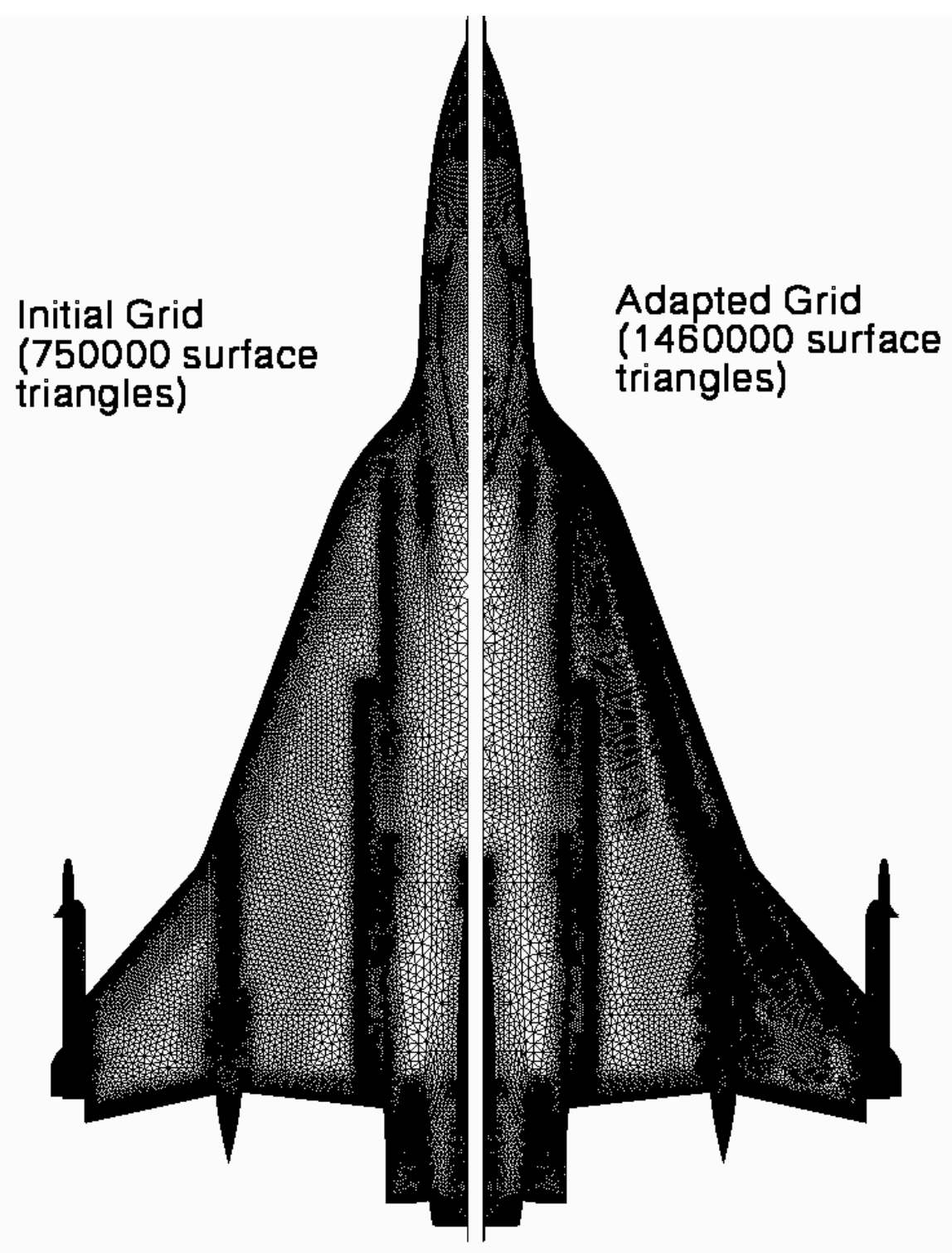

Fig. 12: Initial EADS-MAS surface grid and final adapted grid for flight condition $25\left(\alpha=19.84^{\circ}, M=0.242\right.$ and $\operatorname{Re}=32.2210^{6}$ ). 
This grid has been used as initial grid for all symmetric flight conditions. During the calculations it has been adapted in four steps for each flight condition. In the adaptation feature of the DLR-TAU code the edges of the primary grid are bisected, depending on a refinement sensor. The refinement sensor is based on the differences of the flow variables velocity, density, total pressure and helicity. During the adaptation process, points can be added and removed. The structure of the initial grid, however, is maintained, since only points added to this grid can be removed. The adaptation algorithm can be started after the computation of a flow solution on a certain grid. It then generates a new grid and interpolates the solution onto this grid. The maximum increase of grid points for each of the four adaptation steps was limited to $25 \%$. Grid points have been added in the surface grid and in the tetrahedral grid. The new surface points have been included in the prismatic grid, however the number of the prismatic layers and their thicknesses have not been changed. The initial prismatic layer was designed such that it was suitable for a much finer grid.

Using this adaptation procedure a final adapted grid has been obtained for each flight condition. For example for flight condition 25 the final adapted grid consists of 1,462,096 surface triangles, 32,375,977 prisms, and 25,871,331 tetrahedra. Compared to the initial grid, the number of surface triangles, prisms, and tetrahedra roughly has been doubled, resulting in a total number of 21,149,945 nodes. Fig. 12 shows a comparison of the initial and the final adapted surface grid for flight condition 25 . New grid points mainly have been added along the leading edge (due to the leading edge suction), inboard and outboard of the suction peak of the primary vortex (due to the pressure gradients) and in the tip section of the outer wing. In space, new points have been added in regions with vortical flow above the wing and in the wake region behind the wing.

\section{UTSimCenter}

One of the more unique grid systems has been produced by researchers at the University of Tennessee at Chattanooga SimCenter (UTSimCenter). Two separate grid generation programs have been used to generate the viscous grids used by UTSimCenter within CAWAPI. The first program was a commercially available mesh generation package known as Gridgen. Gridgen was used to create an inviscid unstructured grid. The second grid generation program was developed in-house at the SimCenter and was used to insert viscous layers in the inviscid grid [18]. 


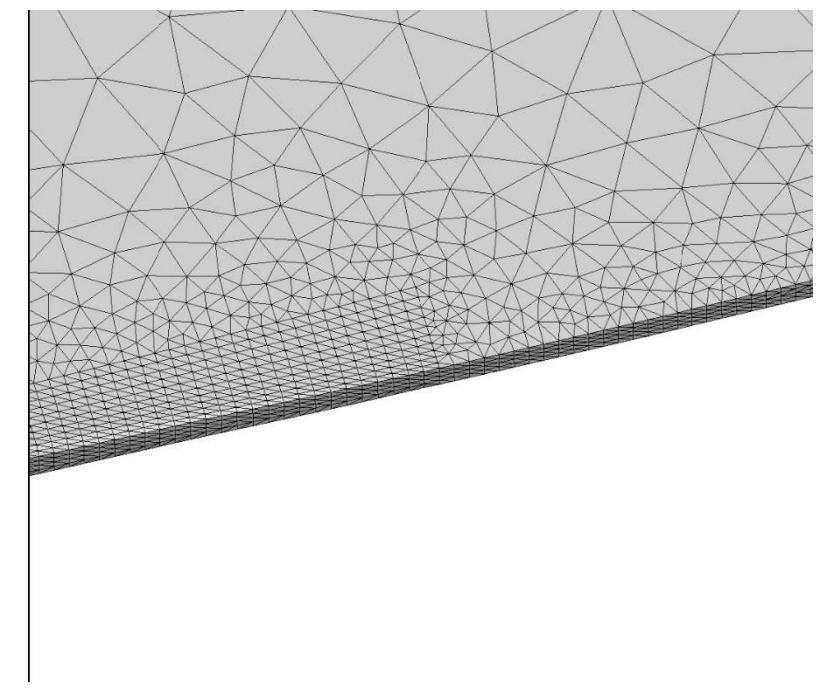

Fig. 13: Leading edge grid showing the converted structured grid domain next to an unstructured grid domain.

\section{Inviscid grid}

Gridgen has been used to create an unstructured inviscid grid, comprising of mostly tetrahedra. A surface grid, consisting of triangular elements, was generated on the air-tight geometry description. Care was taken to ensure proper resolution of pertinent geometric features such as the leading and trailing edge of the wing. The high curvature of the leading and trailing edges needed fine resolution in the chord-wise direction to resolve the shape. The unstructured triangular surface meshing in Gridgen imposes nearly isometric triangular elements. In order to provide the desired resolution in the chord-wise direction and not have an excessive number of elements in the span wise direction a structured grid was used along the leading and trailing edges of the wing. The aspect ratio of the quadrilateral elements was imposed to be no larger than 15 . The resulting structured quadrilateral surface grid was then converted to an unstructured triangular grid by subdividing the quadrilateral elements into two triangles. Fig. 13 shows a section of the leading edge where the converted structured mesh domain meets the unstructured mesh domains. A view of the mesh on the symmetry plane is shown in Fig. 14.

Baffle surfaces were used to control the spacing of the volume grid, resulting in a hybrid unstructured inviscid grid. The quadrilateral elements shown in the figure around the nose and tail are a result of these baffles. Additional baffles were created around the leading edge and trailing edge of the wing and at a near field boundary within a body length of the aircraft. 


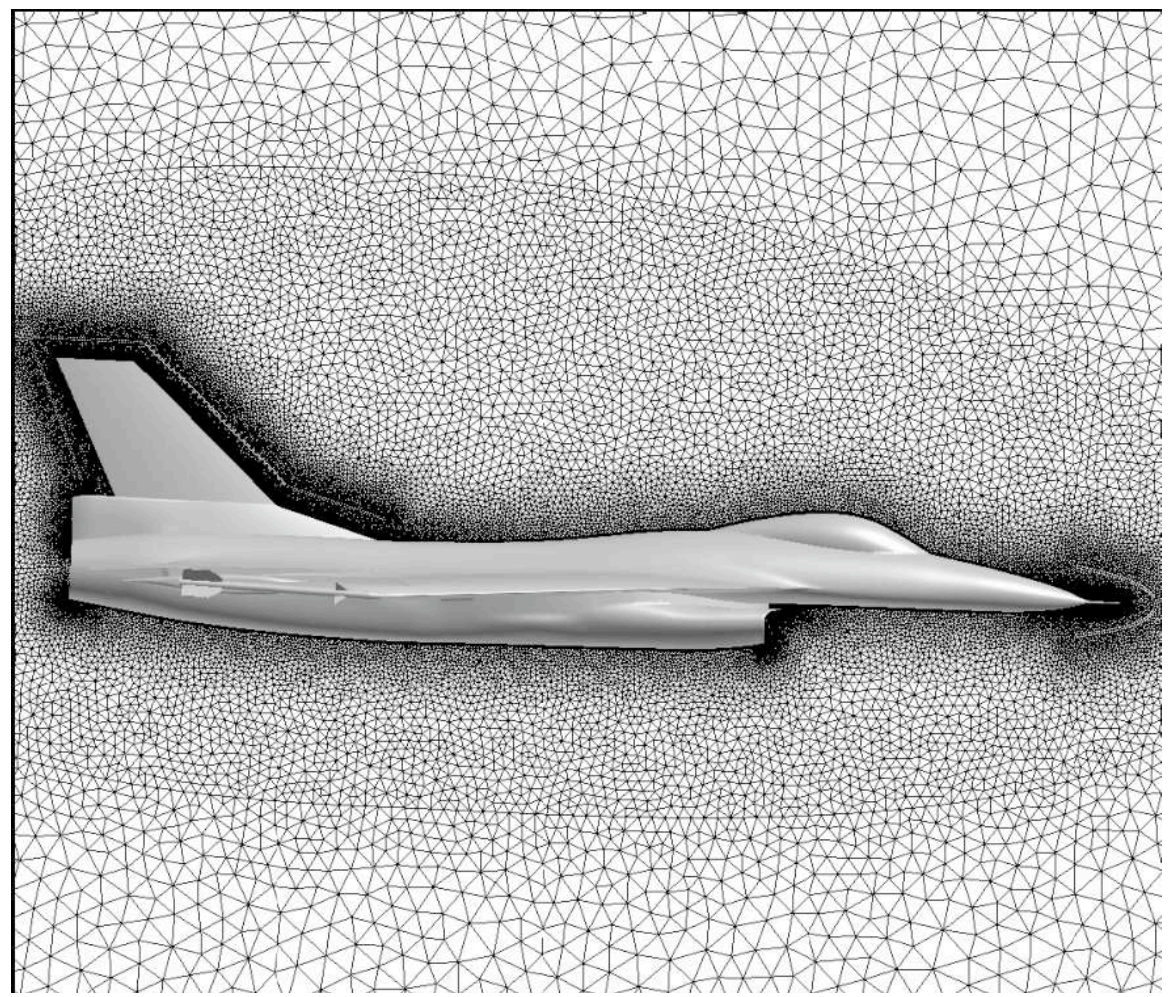

Fig. 14: Symmetry plane of the inviscid unstructured grid generated by UTSimCenter using Gridgen.

\section{Viscous Layer Addition}

An in-house developed grid generation program was used to insert layers of triangular prismatic elements at the no-slip surfaces of the geometry [18]. This method uses a Linear-Elastic grid-smoothing scheme to push the existing grid away from the surface, making room for the viscous elements. The term normally used to define Young's Modulus in the Linear-Elastic relations is defined using a combination of element aspect ratio and corner angles to provide stiffness in regions of tight grid spacing. Poisson's ratio was set to a constant of 0.25 . Only one layer of points is added at a time in reverse order; the top layer is added first and the final layer near the wall is added last. Points are only added where the local mesh spacing is larger than the desired spacing for the current layer. As a result, the number of triangular prismatic elements in a column varies over the surface. Fig. 15 and Fig. 16 show the varying number of elements per column for the grid at the inlet. This capability allows the outer layer of prisms to match the spacing of the local tetrahedral elements without forcing each column to have unnecessary layers, which could result in kinking or buckling of the outer viscous layers. 


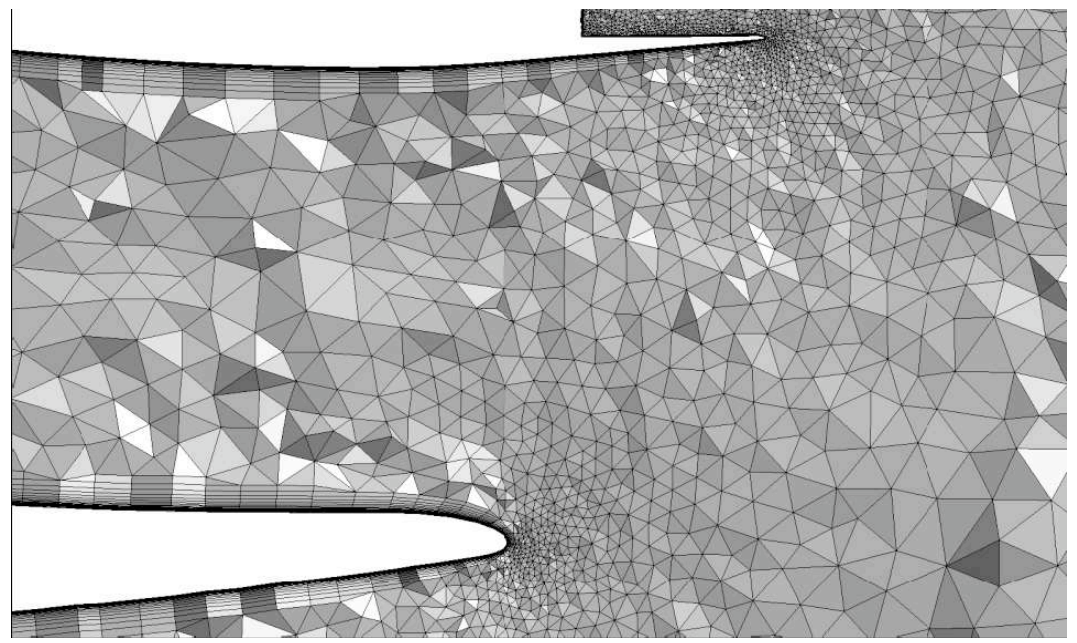

Fig. 15: Wrinkly cutting plane of the viscous unstructured grid generated by UTSimCenter near the symmetry plane at the inlet.

A total of 25 layers were requested for the viscous region. The initial spacing was specified to correspond to an approximate $\mathrm{y}^{+}$-value of one. The height of the subsequent layers increases according to a geometric progression parameter $\Delta \mathrm{s}_{2} / \Delta \mathrm{s}_{1}$ of 1.15 and a geometric growth rate of 1.02. A view of the viscous layers for the tip missile fins is shown in Fig. 17. Finer resolution tetrahedra can be seen in the gap region between the fin and the missile rail. The layer insertion strategy matched the normal spacing of the layers with the existing local tetrahedral grid. The viscous grid for the half-span model of the F-16XL contains 13,906,708 nodes, 32,395,936 tetrahedra, 166,230 pyramid and $15,770,674$ prisms.

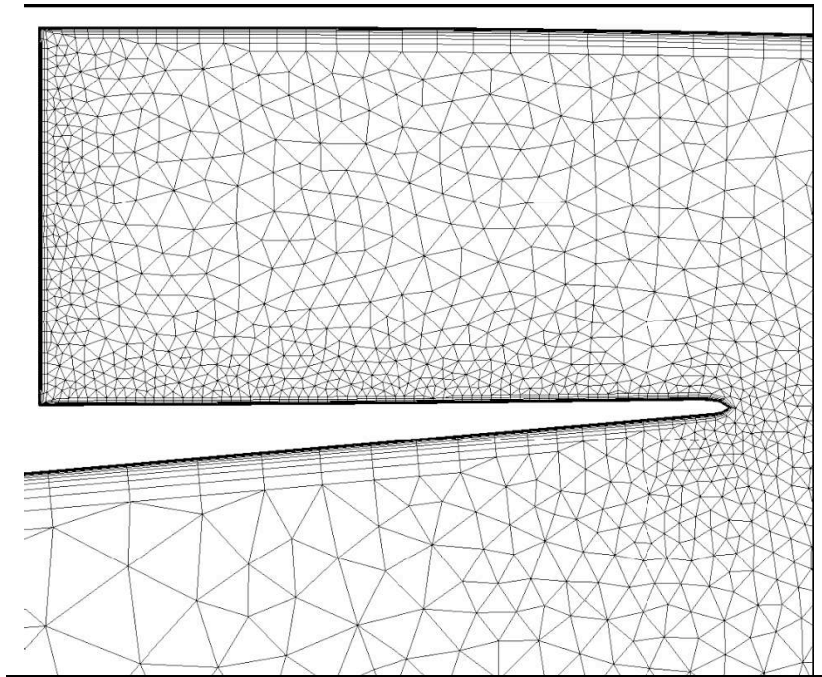

a)

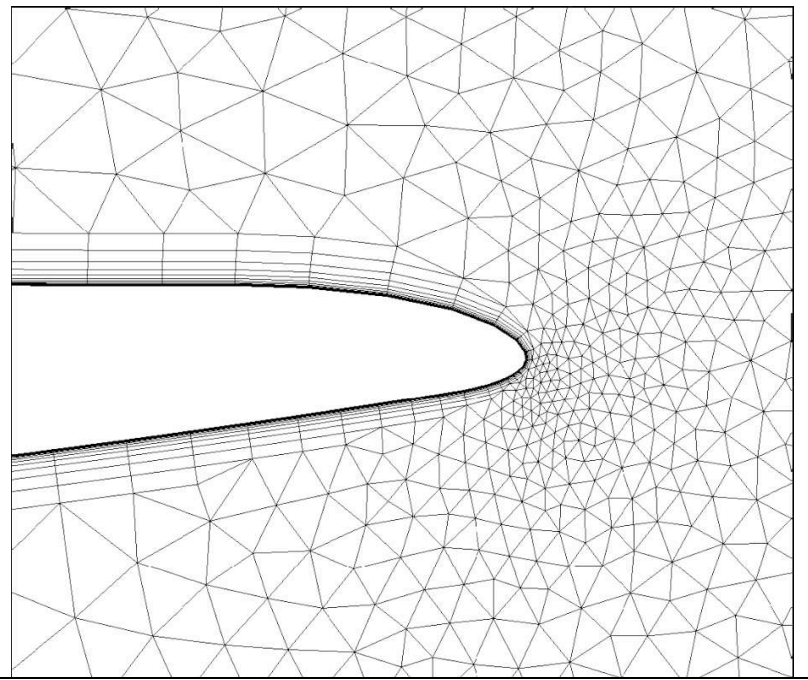

b)

Fig. 16: Magnified views of the grid on the symmetry plane of the viscous unstructured grid generated by UTSimCenter at a) the upper inlet lip and b) the lower inlet lip. 


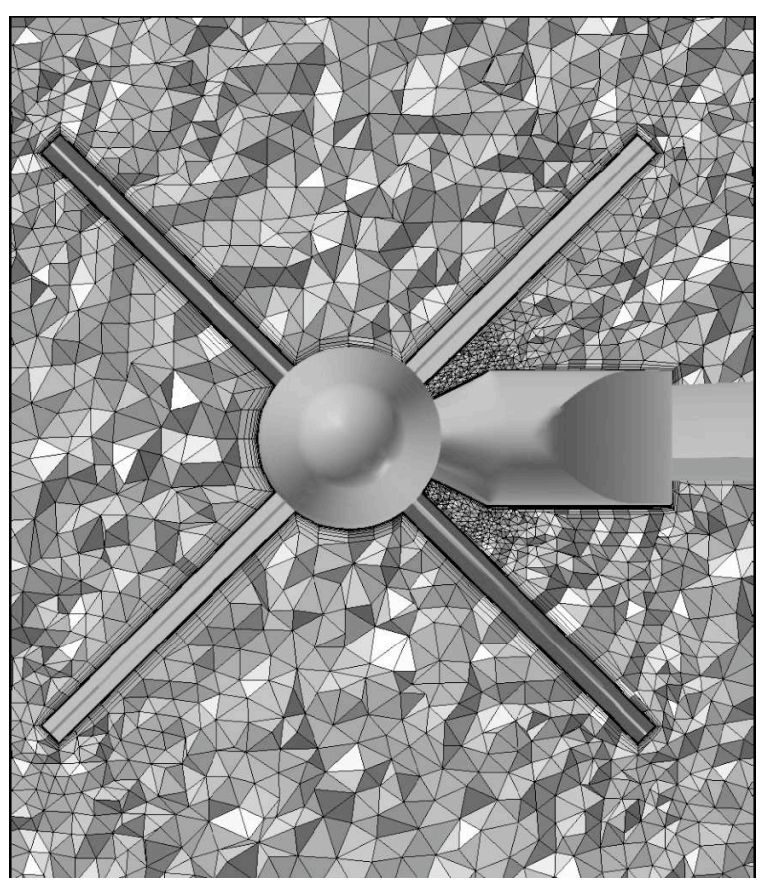

Fig. 17: Magnified view of an axial cut of the viscous unstructured grid generated by UTSimCenter through the tip missile fins and wing.

\section{Boeing Phantom Works}

The grids used by researchers from the Boeing Phantom Works have been generated using the Boeing Modular Aerodynamic Computational Analysis Process (MADCAP). MADCAP was developed at Boeing as a modular framework to house grid generation capabilities from a variety of sources. MADCAP contains a fully automated surface grid generation capability. In addition to the automated approach, the user can interactively control resolution and grid element type through the selection of control nodes, edges and surfaces. Unstructured grid generation algorithms can be selected from Boeing developed libraries and/or from the Advancing Front with Local Reconnection (AFLR) library [19]. Surface grids can contain a combination of quadrilateral and triangular faces. The volume grids used in this study have been developed with the AFLR code using a combination of element types. Near the wall, advancing layers have been used to place highly anisotropic prismatic elements across the boundary layer. Outside the boundary layer, isotropic tetrahedral elements have been utilized. A smooth transition between the prismatic and tetrahedral elements is provided by growing each column of the boundary layer grid until the element at the outside edge is nearly isotropic. The boundary layer resolution is controlled by specification of the initial spacing near the wall, an initial growth rate, a growth stretching and a maximum growth rate. In addition, the 
extent of the boundary layer thickness can be specified or an estimate of the boundary layer thickness for a turbulent flat plate can be used to extend the prismatic layers beyond the estimate. Control of the resolution of the tetrahedral portion of the grid is provided by a linear interpolation from the surface grid. Alternatively, the user can specify a geometry growth rate to control the stretching of resolution in the tetrahedral region. Sources in the form of individual nodes, curves or surfaces can be specified to control the off-body resolution of the tetrahedral grid.

A grid with higher-resolution than the hybrid baseline unstructured grid was constructed in MADCAP to try and improve solution accuracy. In particular, the grid was concentrated near the wing leading edge to try and improve the prediction near the secondary vortex. Grid resolution was increased at the leading edge by introducing high aspect ratio quadrilateral elements into the surface grid. The maximum aspect ratio of the quadrilateral faces is 25 . The circumferential resolution at the leading edge is $1.5 \times 10^{-4} \mathrm{c}_{\mathrm{r}}$ inboard of the wing crank transitioning to $3.0 \times 10^{-5} \mathrm{c}_{\mathrm{r}}$ spacing near the wing tip. The quadrilateral elements were subdivided into triangles in the final grid. A comparison of the Boeing and common hybrid baseline unstructured grid at the wing leading edge is shown in Fig. 18. The resolution of the Boeing surface grid is about double that of the common mesh in the immediate proximity of the wing vortices.

The volume portion of the Boeing grid was generated in AFLR and consists of a semi-structured boundary layer extrusion connected to an isotropic tetrahedral grid. The extrusion used a $9.1 \times 10^{-7} c_{r}$ initial spacing at the wall to yield a $\mathrm{y}^{+}$-value of approximately 1 . The initial spacing grew geometrically with an initial geometric progression parameter $\Delta \mathrm{s}_{2} / \Delta \mathrm{s}_{1}$ of 1.2 ending at a 1.8 maximum growth ratio. Extrusion terminated when the prisms achieved an aspect ratio near unity. The combination of the initial viscous spacing, growth rate parameters, and surface spacing produced approximately 15 prism layers. The resulting volume grid had 19.3 million cells.

The tetrahedral portion of the Boeing grid has been refined at flight conditions 7 and 25 using feature-based grid adaption. 


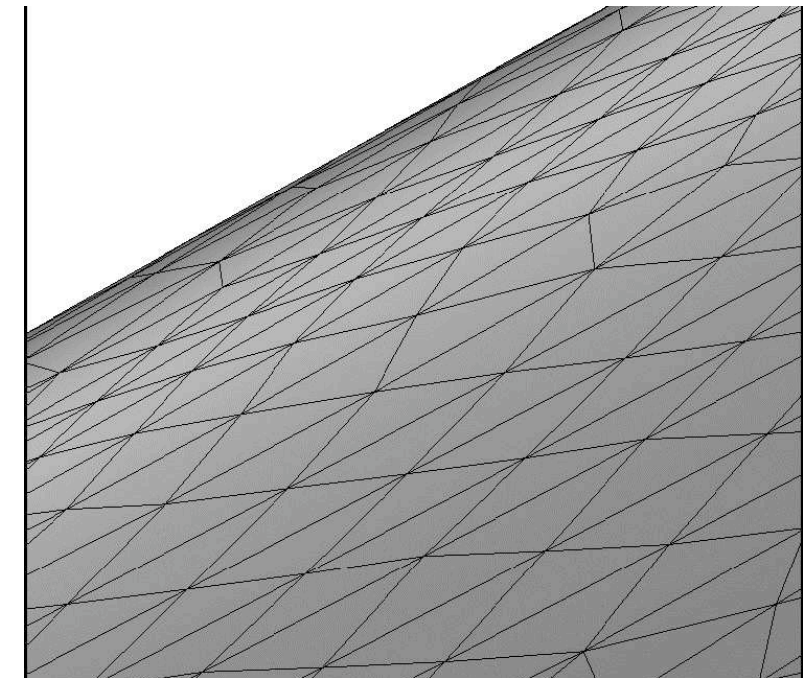

a)

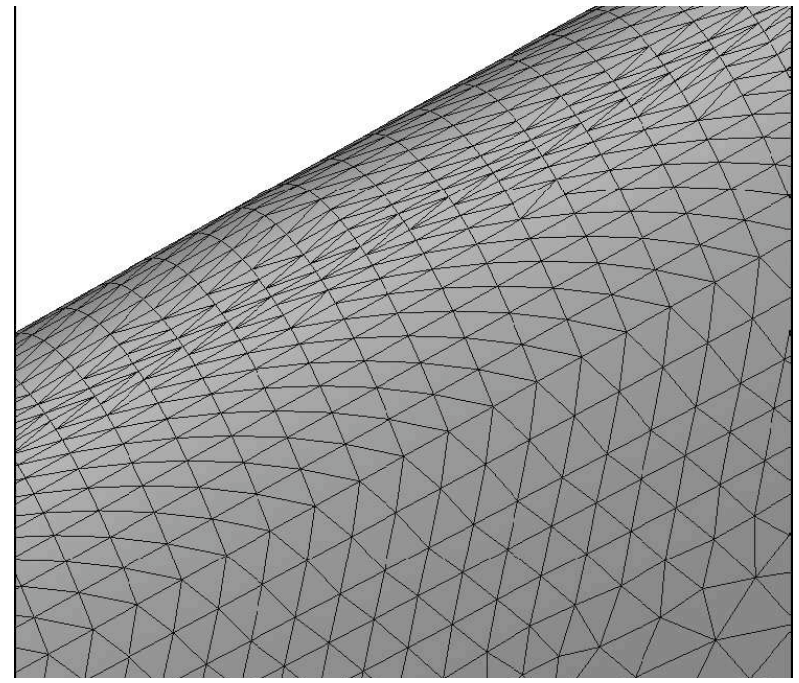

b)

Fig. 18: Comparison of surface grids near the wing leading edge: a) hybrid baseline unstructured grid and b) Boeing grid.

\section{Conclusion}

In the framework of the Cranked-Arrow Wing Aerodynamics Project International (CAWAPI) both structured and unstructured grids have been generated (see Table 1).. Prior to the grid generation an IGES file containing the air-tight geometry description of the half-span model of the F-16XL aircraft that could be used for both the structured and unstructured grid generation has been generated.

The baseline structured grid has been generated by NLR using in-house developed (semi-automatic) grid generation algorithms. A family of grids including grids with a reduced number of blocks have been derived from this baseline grid. Although most of the algorithms used had become available just before CAWAPI and thus only a limited experience with their application to such a complex configuration as the F-16XL aircraft had been gained, a grid of good quality was generated well within four weeks, including some further development of the grid generation algorithms This time compared favourably with that required to produce the unstructured grids in CAWAPI. The best practices established during CAWAPI have resulted in a significant reduction of the structured grid generation time for future projects.

Several unstructured grids have been generated within CAWAPI. The baseline all-tetrahedral and hybrid unstructured grids have been generated at NASA Langley Research Center and USAFA, respectively. Despite their rather moderate cell count, the baseline all-tetrahedral and hybrid unstructured grids provided sufficient geometrical 
resolution. However, several CAWAPI members needed grids with more geometrical resolution. Trimmed unstructured grids have been generated at EADS-MAS, the UTSimCenter, Boeing Phantom Works and KTH/FOI.

Both results obtained on the structured grids and the unstructured grids showed a significant improvement in agreement with flight test data in comparison with those obtained on the structured multi-block grid used during CAWAP [1][3]. The CAWAPI results obtained on the structured grids are described and discussed in Refs. [4] and [7], whereas the results obtained on the unstructured grids are detailed in Refs. [5]-[7].

\section{Acknowledgments}

The authors gratefully acknowledge the support provided by Lockheed Martin Aeronautics Company - Fort Worth in providing the refined iges geometry file. In addition, the authors gratefully acknowledges the geometrical work performed by Mr. Edward B. Parlette of Vigyan, Inc. in generating unstructured grids from the iges file.

Part of this work has been conducted under NLR's programmatic research funding.

\section{References}

[1] Lamar, J.E., Obara, C.J., Fisher, B.D, and Fisher, D.F., "Flight, wind-tunnel, and Computational Fluid Dynamics Comparison for Cranked Arrow Wing (F-16XL-1) at Subsonic and Transonic Speeds,” NASA TP-2001-210629, 2001.

[2] Hummel, D., and Redeker, G., “A New Vortex Flow Experiment for Computer Code Validation,” RTO-AVT Symposium on Advanced Flow Management; Part A - Vortex Flow and High Angle of Attack Papers on Disc [CDROM], Paper Number 8, May, 2001.

[3] Obara, C.J.; and Lamar, J.E., "Overview of the Cranked-Arrow Wing Aerodynamics Project International," AIAA Journal of Aircraft, published online xx xxx. 2007. doi: xx.xxx/x.xxxxx

[4] Boelens, O.J., Badcock, K.J., Elmilgui, A., Abdol-Hamid, K.S., and Massey, S.J., "Comparison of Measured and Block Structured Simulations for the F-16XL Aircraft," AIAA Journal of Aircraft, published online xx xxx. 2008. doi: $\mathrm{xx} \cdot \operatorname{xxx} / \mathrm{X} \cdot \mathrm{xxxxx}$

[5] Goertz, S., Jirasek, A., Morton, S.A., McDaniels, D.R., Cummings, R.M.; Lamar, J.E., and Abdol-Hamid, K.S., “Standard Unstructured Grid Solutions for CAWAPI F-16XL," AIAA Journal of Aircraft, published online xx xxx. 2008. doi: xx.xxx/X.xxxxx

[6] Fritz, W., Davis, M.B., Karman, S.L., Jr., and Michal, T., "RANS Solutions for the CAWAPI F-16XL Using Different Hybrid Grids," AIAA Journal of Aircraft, published online xx xxx. 2008. doi: xx.xxx/x.xxxxx 
[7] Rizzi, A., Jirasek, A., Badcock, K.J., Lamar, J.E., Boelens, O.J., and Crippa, S., "What Was Learned from Numerical Simulations of F-16XL (CAWAPI) at Flight Conditions," AIAA Journal of Aircraft, published online xx xxx. 2008. doi: xx.xxx/x.xxxxx

[8] Lamar, John E.; Cronin, Catherine K.; and Scott, Laura E., "Virtual Laboratory Enabling Collaborative Research in Applied Vehicle Technologies," RTO-AVT Symposium on "Flow Induced Unsteady Loads and The Impact on Military Applications Papers on Disc [CD-ROM], Keynote 2, April, 2005.

[9] Boerstoel, J.W., Kassies, A., Kok, J.C., and Spekreijse. S.P., "ENFLOW, a Full-Functionality System of CFD Codes for Industrial Euler/Navier-Stokes Flow Computations,” NLR TP 96286U, NLR, Amsterdam, 1996.

[10] Spekreijse, S.P., Prananta, B.B. and Kok, J. C., “A Simple, Robust and Fast Algorithm to Compute Deformations of Multi-Block Structured Grids,” NLR-TP-2002-105, NLR, Amsterdam, 2002.

[11] Legensky, S. M., Edwards D. E., Bush R. H., Poirier D. M. A., Rumsey, C. L., Cosner R. R., and Towne, C. E., “CFD General Notation System (CGNS): Status and Future Directions,” AIAA Paper 2002-0752, 2002.

[12] Samareh, S., “GridTool: A Surface Modeling and Grid Generation Tool,” NASA CP-3291, 1995.

[13] Pirzadeh, S., "Progress toward a User-Oriented Unstructured Viscous Grid Generator," AIAA Pape 96-0031, 1996.

[14] Strang, W. Z., Tomaro, R. F., and Grismer, M. J., "The Defining Methods of Cobalt: A Parallel, Implicit, Unstructured Euler/Navier-Stokes Flow Solver,” AIAA Paper 99-0786, 1999.

[15] Wallin, S., "Standardized Data Format, The Aeronautical Research Institute of Sweden (FFA)," FFAP-A-950, Stockholm, Sweden, 1992.

[16] Eliasson, P., “EDGE, a Navier-Stokes Solver for Unstructured Grids”, Proc. To Finite Volumes for Complex Applications III, ISBN 190399634 1, pp. 527-534, 2002.

[17] Gerhold, T., Friedrich, O., Evans, J., Galle, M., "Calculation of Complex Three-Dimensional Configurations Employing the DLR-Tau Code,” AIAA Paper 97-0167, 1997.

[18] Karman, S. Jr., "Unstructured Viscous Layer Insertion Using Linear-Elastic Smoothing,” AIAA Paper 2006-0531, 2006.

[19] Marcum, D. L., “Advancing-Front/Local-Reconnection (AFLR) Unstructured Grid Generation,” Computational Fluid Dynamics Review, World Scientific, Singapore, 1998, p. 140. 